\title{
Optical polarization rogue waves in fiber laser
}

\author{
Lei Gao ${ }^{1}$, Tao Zhu', ${ }^{1, *}$, Stefan Wabnitz ${ }^{2}$, Min Liu', and Wei Huang ${ }^{1}$ \\ ${ }^{1}$ Key Laboratory of Optoelectronic Technology \& Systems (Ministry of Education), Chongqing University, Chongqing 400044, China. \\ ${ }^{2}$ Dipartimento di Ingegneria dell'Informazione, Università degli Studi di Brescia and INO-CNR, via Branze 38, 25123 Brescia, Italy. \\ Authore-mail address: zhutao@cqu.edu.cn
}

\begin{abstract}
A new kind of optical rogue waves, polarization rogue waves that appear with greatly deviated and unpredictable positions of polarization states, is identified in forming partially modelocked fiber laser based on parametric frequency conversion.

OCIS codes: (190.0190) Nonlinear optics; 190.4410 Nonlinear optics, parametric processes; (190.3100) Instabilities and chaos (260.5430) Polarization
\end{abstract}

\section{Introduction}

Optical rogue waves (RW) that occur with unexpectedly probability have been frequently reported in various fields, such as supercontinuum, fiber laser, semiconductor laser, and femtosecond filamentation. L-shaped probability has been observed either in temporal or spectral domains. Those two kinds of RWs are equivalent due to Fourier transformation. Here, we demonstrate a new kind of RW, depending on the state of polarization (SOP) of the generated frequencies in forming partially mode-locked fiber laser (PML), in a new pattern formation process, where the laser redistributes its energy from center wavelength to sidebands through parametric instability (PI), and subsequently longitudinal modes are populated via cascaded four-wave-mixing (FWM). Experimental results prove that the PI, which exhibits great fluctuations when it is spontaneously grown from noise in conservative systems [2], possess a high degree of coherence in dissipative system. Specifically, we find that the SOPs of the frequencies generated by cascaded FWM shows a tendency from fixed state to bifurcation, and finally to randomization. In this process, we define a new kind of RW, the optical polarization rogue waves (PRW), as they appear with positions deviating from the mean positions, and the SOP of the generated frequencies are totally unpredictable.

\section{Experimental results}

The dispersion-managed fiber ring cavity consists of $1 \mathrm{~m}$ erbium-doped fiber, $19.5 \mathrm{~m}$ dispersion compensation fiber, $14.5 \mathrm{~m}$ single mode fiber with dispersions of $15.7,-38$, and $18 \mathrm{ps} / \mathrm{nm} / \mathrm{km}$, respectively (more details are in Ref. 4). A saturable absorber is utilized to detuning nonlinear phase for phase matching through adjusting the SOP of light. The presence of periodically varying dispersion and loss/gain permits parametric instability (PI) to grow when the condition for quasi-phase-matching is satisfied. Physically, the generation of PML is similar to the generation of chaotic Kerr combs based on parametric frequency conversion within high quality factor (Q) microresonators. Primarily, energy transfers from a continuous wave $(\mathrm{CW})$ central mode into parametric or MI gain sidebands: corresponding longitudinal modes are thus populated. Next, cascaded FWM may occur among the pump and different MI gain lobes. When the pump power is high enough, newly generated frequencies merge into a gap-free or continuum comb. This process is illustrated in Fig. 1, where two vector FWM processes are shown.

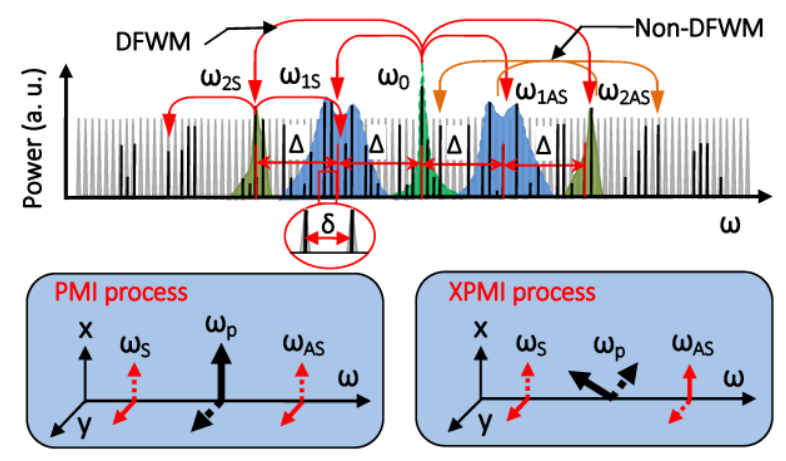

Figure 1. PML dynamics based on stochastic parametric conversion. The insets are phase-matching processes of two different vector MI processes. The solid and dotted lines represent two orthogonal polarization components, respectively.

We filtered out the PML spectrum by a wavelength tunable filter with a bandwidth of $1 \mathrm{~nm}$, and find that different sets of longitudinal modes correspond to different portions of the pulse packets. It is clear that the center wavelength region is dominated by self-phase modulation, and for wavelengths far away from the center, the number of pulses 
in each packet decreases. Besides, we measure the SOP of the filtered spectrum, and an evolution process of the SOP shown in Fig. 2 indicates that the cascaded FWMs among high coherent PI sidebands introduce significant fluctuations of the SOPs.
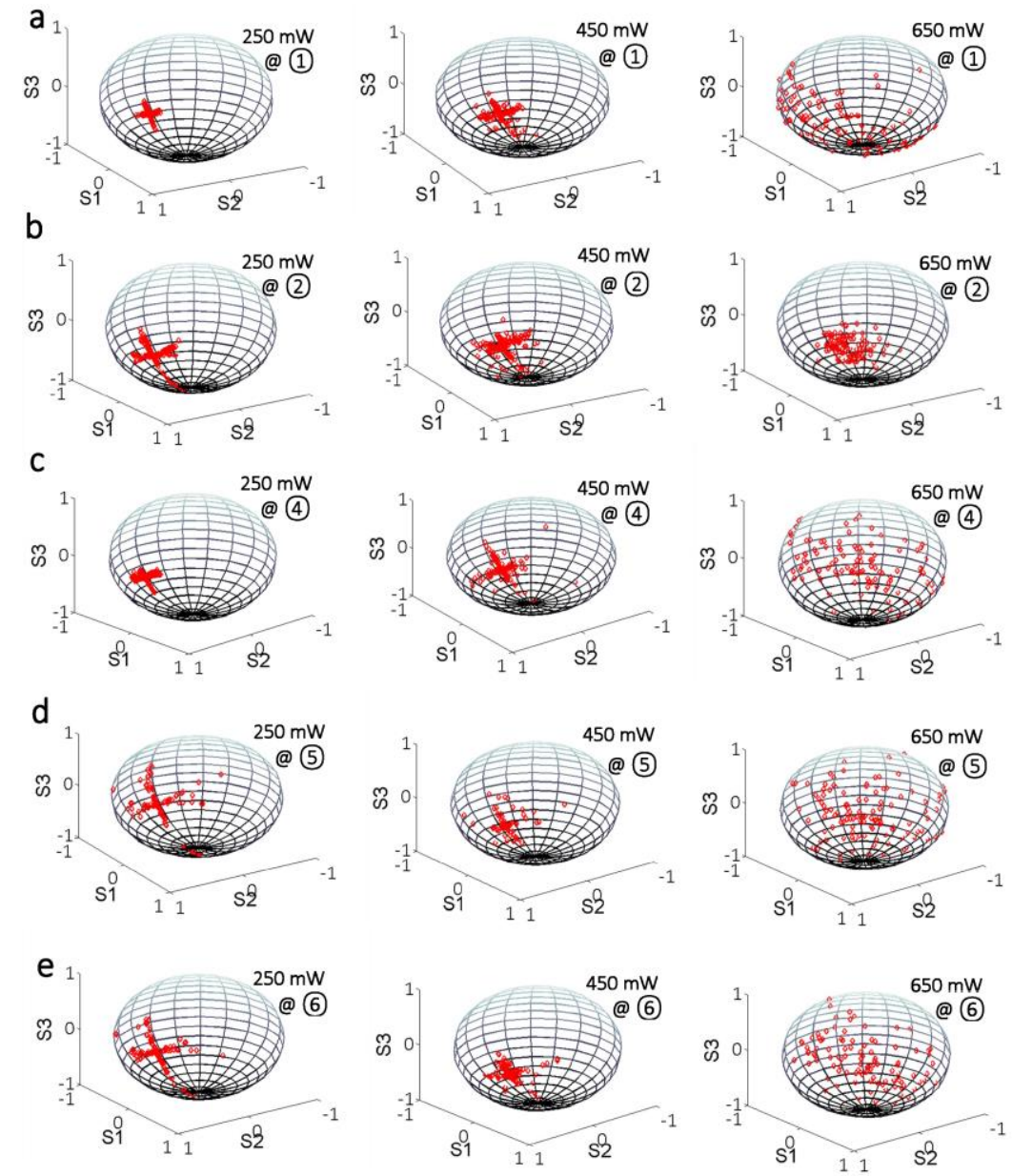

Figure 2. Experimentally measured SOPs for filtered wavelengths under various pump powers. The wavelengths of (1), (2), (4), (5), (6) are $1547.6 \mathrm{~nm}, 1556.1 \mathrm{~nm}, 1565 \mathrm{~nm}, 1574.1 \mathrm{~nm}$, and $1582.7 \mathrm{~nm}$, respectively. Those wavelengths are spanning in the broad spectrum of PML.

Moreover, we identify significant temporal and spectral RWs in this formation process, and a new kind of RW, polarization RW is also observed in Fig. 2. Primarily, the SOP of the center wavelength remains a fixed point on the Poincaré sphere, while wavelengths far away from the center mode bifurcate into a cross-like configuration of output SOPs. Besides, irregular polarization states located outside of the main polarization directions emerge for red-shifted sidebands whenever the pump power reaches $250 \mathrm{~mW}$, and SOP scattering aggravates over the whole wavelength span for the pump power of $450 \mathrm{~mW}$. We attribute the polarization deviation from the cross shape to chaotic competition of cascaded FWM processes, including both scalar and vector FWMs, through which the energy of longitudinal modes with a SOP along the cross is transferred into newly-generated SOPs. When the pump power is larger than $600 \mathrm{~mW}$, the SOPs of filtered wavelengths appear as totally random, owing to enhanced spectral energy transfer processes. During this processes, the quasi-phase-matching may be varied as the nonlinear phase response of the SA are power-dependent, and significant phase accumulation triggers the emergence of RW when cascade FWMs occur.

For the dissipative characteristic of the observed phenomenon here, the conservative nature of regular single or coupled nonlinear Schrödinger equations cannot predict a good description. Therefore, a coupled Ginsberg-Landau equations with dissipative terms are needed to fully explain the whole process.

\section{References}

[1] J. M. Dudley, F. Dias, M. Erkintalo, and G. Genty, "Instabilities, breathers and rogue waves in optics," Nat. Photon., 8, 755-764 (2014).

[2] D. R. Solli, G. Herink, B. Jalali, and C. Ropers, "Fluctuations and correlations in modulation instability," Nat. Photon., 6, 463-468 (2012).

[3] L. Gao, T. Zhu, S. Wabnitz, M. Liu, and W. Huang, "Coherence loss of partially mode-locked fiber laser," arXiv, 1410.3228 (2016). 